\title{
Survey on demand of the aged people for college volunteers in home nursing care service
}

\author{
Meng Tian, Wenjuan Zhong ${ }^{\mathrm{a}}$ and Jia Guo \\ Health Science and Nursing College of Wuhan Polytechnic University, Hubei 430023 China
}

\begin{abstract}
Objects: To study the demand of the aged community residents for college volunteers in home nursing care service and its influence factors. Methods: Questionnaires on the aged residents' expectation and requirements for college volunteers in home nursing care was prepared on basis of studies of reference books, study materials and pre-investigation, and then the questionnaires was completed by means of convenient sampling of 200 aged residents. Results The demand and expectation of the elderly for college volunteers in health care and spiritual comfort was strong. The old people who have more children, living alone and often feel lonely are more likely to need college volunteers. Conclusions: Volunteers should be arranged reasonably according to the needs of the aged people, and priority should be given to those who have more children, living alone and often feel lonely.
\end{abstract}

\section{Introduction}

Home nursing care service is the key to solve the pension problem in China and also the direction for future development of China's pension. Home nursing care service refers to the government and social communities arrange care workers with basic nursing skills to provide home nursing care services for the aged, including daily life care, housekeeping service, rehabilitation nursing and spiritual comfort services. More researches have been made on home care services, but not much in respect of home care service provider. Some scholars believe that home care solely provided by the government is far beyond adequacy, we should give full play to the market, private non-enterprise units and social workers [1-3]. Several scholars point out that, based on qualitative analysis on home care, it is imperative to introduce volunteers[4-6]. Voluntary activity is a kind of selfless activity contributed by individuals or a group of people in absence of financial or material benefits with the purpose to improve the quality of life of human beings [7]. Home care for the aged people provided by the volunteers for free, thus the community can save money for the more difficult groups. As a result, more old people can access and enjoy home care service[8]. This research is conducted to investigate the needs of the elderly for college volunteers to participate in home care services. The details are as follows.

\footnotetext{
${ }^{a}$ Corresponding author: wjzhong8616@126.com

The content of this essay is one of the achievements in relation to philosophy and social science from major research project Interactive Research on Community Development and College Education by Hubei Provincial Department of Education, the item number is $2015 \mathrm{z} 33$
} 


\section{Object and Method}

\subsection{Object of research}

The object of research was chosen from four residential communities of Changqing Garden of Wuhan. Standard: (a) above 60 years old; (b) aware and easy to communicate with; (c) volunteer to be the object of the research. Exclusion: (a) below 60 years old; (b) unconsciousness or difficult to communicate; (c) unwilling to be the object of the research.

\subsection{Method of study}

The purpose and significance of the investigation were explained by trained investigators so as to obtain their consent and cooperation. The questionnaires were filled by the object of the research independently, and the researchers filled the questionnaires for those who have difficulty in writing according to their answer. Totally 200 questionnaires were handed out and 195 valid questionnaires were collected back, $97.5 \%$ valid.

\subsection{Means of research}

The items in the questionnaire were drawn up in reference to a large amount of research papers and work as well as pre-investigation among the object group of people, which include: (a) general information of the aged people, such as gender, age, marital status, educational level, occupation, living independence, economic conditions, relationship with family members, caregivers, feeling of loneliness and so on; (b) the needs for college volunteers: including life care, medical care, spiritual comfort and legal consulting; (c) other: degree of expectation of volunteers for home care and positive aspects of the volunteers, etc..

\subsection{Statistical Methods}

A database was created by using SPSS22.0 software to make statistical description over the data collected and the independence of the qualitative data was proved by means of chi square test. The factors that affect the choice will of the aged people were analyzed by means of two element regression analysis, and the statistical data was useful in case $\mathrm{P}<0.05$ for variance.

\section{Results}

\subsection{General information of the aged people}

The age of people subject to the research ranged from 60 to 96 years old, or 8.5 years above or below 76.5. And the general information of the aged group was shown as follows. Gender: 74 males; 121 females. Marital status: 110 live with spouses; 2 divorced; 83 bereft of their spouses. Education level: 59 of or below primary school; 99 of high school or secondary specialized school; 32 of college or above. Occupation before retirement: 20 farmers; 110 enterprise employees; 46 public institutional staff; 19 others. Living independence: 153 live independently; 42 partially-independent. Status of economics: 60 live with surplus money; 100 live with roughly enough money; 34 live with slight economic difficulty; 1 live with economic difficulty. Payment of medical treatment: 186 covered by medical insurance; 7 pay by themselves or their spouses; 2 pay by their children. Children: 1 without child; 34 with one child each; 70 with two children each; over 80 with three or more children. Way of living: 48 live alone; 76 living with spouse; 63 live with children; 8 live in pension institutions; Whether have caregivers: 54 unattended, 81 persons by their spouses, 56 persons by children, 4 persons by other ways; Feelings of loneliness: often 30 people, occasionally 69 people, 
never 97.

\subsection{The needs of the aged people for college volunteer in respect of home care}

Among expected services by college volunteers, medical care or nursing was up to $36.1 \%$ in proportion, while spiritual comfort $33.33 \%$, daily living care $20.8 \%$ and legal consulting $9.8 \%$.

\subsubsection{The need of the aged people for college volunteers in respect of medical care}

The need for health lecture was of highest proportion of $55.9 \%$, followed by rehabilitation training $51.3 \%$, regular physical examination $37.9 \%$, emergency relief $34.9 \%$, and accompany to see a doctor $30.7 \%$.

\subsubsection{The need of the aged people for college volunteers in respect of spiritual comforts}

Art Gala (60\%) accounted for the highest in need, followed by tea Forum (54.4\%), calligraphy and painting contest $(33.3 \%)$, chat to relieve boredom $(31.8 \%)$, psychological counseling (18\%) and reading $(7.2 \%)$.

\subsubsection{The aged people's demand for college volunteers in life care}

Housekeeping (40\%) accounted for the highest in proportion of demand, followed by food delivery (30.8\%), wash clothes (18.5\%), shopping (15.4\%), pick out (13.3\%) and bath $(3.6 \%)$.

\subsubsection{The need of the aged people for college volunteers in respect of law service}

Legal assistance was of $19.5 \%$, legal advice $17.9 \%$, legal lectures $13.4 \%$.

\subsection{Influential factors of the aged people's demand for college volunteers to participate in home nursing care service}

3.3.1 Single factor analysis on the aged people's demand for college volunteers to participate in home nursing care service

The research indicated that factors affecting the aged people's demand for college volunteers to participate in home nursing care include: ways of living, number of children, loneliness, self-care ability in daily life, with their discrepancies having statistical significance. Details are shown in Table 1 as following.

Table 1. Single Factor Analysis on the Demand of the Aged people's for College Volunteers to Participate in Home Nursing Care Service( $\mathrm{n}=195)$

\begin{tabular}{llllll}
\hline Item & & wish(\%) & Not wish(\%) & $\chi^{2}$ & P \\
\hline number of children & 1 & $21(61.8)$ & $13(38.2)$ & & \\
& 2 & $60(85.7)$ & $10(14.2)$ & 13.841 & 0.008 \\
3 & $46(90.2)$ & $5(9.8)$ & & \\
Ways of living & Above 3 & $34(87.2)$ & $5(12.8)$ & & \\
& Alone & $47(97.9)$ & $1(2.1)$ & & \\
& With spouse & $58(76.3)$ & $18(23.7)$ & 16.585 & 0.001 \\
\hline
\end{tabular}




\begin{tabular}{|c|c|c|c|c|c|}
\hline & With children & $53(84.1)$ & $10(15.9)$ & & \\
\hline & $\begin{array}{l}\text { Endowment } \\
\text { inst. }\end{array}$ & $4(50)$ & $4(50)$ & & \\
\hline \multirow[t]{3}{*}{ Loneliness } & Often & $29(96.7)$ & $1(3.3)$ & 11.471 & 0.003 \\
\hline & Seldom & $61(89.7)$ & $7(10.3)$ & & \\
\hline & Never & $72(74.2)$ & $25(25.8)$ & & \\
\hline \multirow[t]{2}{*}{ Self-care ability } & entirely & $121(79.1)$ & $32(20.9)$ & 8.052 & 0.002 \\
\hline & partially & $41(96.7)$ & $1(2.4)$ & & \\
\hline
\end{tabular}

\subsubsection{Binary logistic analysis on the aged people's demand for college volunteers to participate in home nursing care service}

Taking whether the aged people's wish college volunteers to participate in the home nursing care as dependent variable and ages, gender, marital status, number of children, ways of living, loneliness, self-care ability in daily life, educational background as independent variable (assignment of number of children: 1 child $=0,2$ children $=1,3$ children $=2$, above 3 children=3; assignment of ways of living: living alone $=0$, living with spouse $=1$, living with children $=2$, live in endowment institutions $=3$; assignment of loneliness: often $=0$, seldom $=1$, never $=2$ ), binary logistic analysis was carried out with 3 variables in this binary regression equation. The results showed that the elderly who have more children, live alone and often feel lonely are more likely to need college volunteers. For details, please refer to the following Table 2 .

Table 2. Binary Logistic Analysis on the Aged People's Demand for College Volunteers to Participate in Home Nursing Care Service $(\mathrm{n}=195)$

\begin{tabular}{|c|c|c|c|c|c|c|}
\hline Item & B & S.E. & Wald & $\mathrm{df}$ & Significance & $\operatorname{Exp}(B)$ \\
\hline Number of children & 0.519 & 0.214 & 5.863 & 1 & 0.015 & 1.681 \\
\hline Ways of living & -0.644 & 0.231 & 7.755 & 1 & 0.005 & 0.525 \\
\hline Loneliness & -1.039 & 0.374 & 7.722 & 1 & 0.005 & 0.354 \\
\hline Constant value & 4.041 & 1.345 & 9.031 & 1 & 0.003 & 56.886 \\
\hline
\end{tabular}

\section{Discussion}

The aged people are in great demand for volunteers in health care and spiritual comfort, which is in line with the research results concluded by Guo Guanjun[9], Gao Jing[10], etc. It can be seen that the aged people became more aware of health care along with the improved living standard of people. The research indicated that the aged people had more demand for college volunteers to provide health knowledge explanation and rehabilitation exercises in respect of health care, almost the same with the research results made by Zhao Rong[11]. However, the demand for periodic physical examination, first aid service, seeing doctor accompanied by someone was not so high, which is probably due to the fact that most of the aged people are covered by medical care insurance and have access to periodic physical examination every year. Meantime, affected by traditional ideas, many aged people prefer looking doctor accompanied by their family members and obtaining help by acquaintance surroundings in case of emergency. Furthermore, as the decline of body function, partial loss of social function and narrow social relationship circle, the aged people yearn for college volunteers to conduct some recreational activities to enrich their life. Regarding spiritual comfort, the aged people have higher demand for arts festival and tea party, while have lower demand for chatting and psychological consultation, which are possibly due to the fact that $78.5 \%$ of the aged people participating in this questionnaire can entirely look after themselves, can communicate with people surrounding them and 
thus have little interest in chatting with college volunteers who have generation gap with the aged people.

The aged people who have more children, live alone and often feel lonely are more likely to need college volunteers. The self-care ability was not in the regression equation, but the single factor analysis indicated that it had connection with the aged people's demand for college volunteers. Study conducted by Xing Fengmei[12] also suggested that self-care ability is one of the reasons why the aged people need home nursing care service. From our point of view, $78.5 \%$ of the elderly participating in this questionnaire can entirely look after themselves, only a few of the elderly are people who have partial self-care ability and have no self-care ability at all. Therefore, we should also consider the self-care ability. The aged people who have more children are more eager to receive home nursing care by college volunteers, which is probably due to the fact that it is widely believed the more children the aged people have, the more care the aged people received from their children thus they have little need for college volunteers. However, the survey conducted by Chen Zhike[13] indicates that the hypothesis of "the fewer children the aged people have, the more demand they have for home nursing care" failed in statistics verification $(\mathrm{P}=0.884)$. We view that the more children the aged people have, the easier the custody dispute brought, the more home nursing care services are wanted: children themselves would shuffle off the custody responsibility for their parents and the elderly could not be taken good care. Given this, further study is needed.

Compared with other aged people, those who live alone and often feel lonely are more eager for college volunteers for home nursing care, which is in conformity with the research result conducted by McCallion[14]. Besides, the survey also indicated that $79.5 \%$ of the elderly wish college volunteers to participate in the home nursing care service. As you see, college volunteers play a non-negligible role in home nursing care service, especially for the aged people who have more children, live alone and often feel lonely. Among the home care services by college volunteers, the top three the aged people valued most are service attitude, specialized knowledge, mainly means medical science knowledge and duty on time. Therefore, training relating to those three aspects should be enhanced to college volunteers.

The aged people's need for daily life care is lower than that of medical care and spiritual comfort. The survey conducted by Wu Jialin[15] shows that daily life care by their family member is more satisfactory than that of volunteers. We viewed this as one of the reasons why the aged people have little demand for volunteers and $78.5 \%$ of the aged people in this questionnaire are those can look after themselves entirely.

It was found through the survey that the aged people have little demand for legal advice, which is probably because the aged people seldom encounter legal disputes in their daily life.

\section{Conclusions}

To make a long story short, the demand by the aged people for college volunteers in home nursing care is great, especially for medical health care and spiritual comfort. Number of children, ways of living and loneliness are the main factors affecting demand of the aged people for college volunteers to participate in home nursing care service. In view above, the community can arrange college volunteers nearby to provide regular service for the aged people and give priority to those who have more children, live alone and often feel lonely. To work out guideline and policy for college volunteers in home nursing care, a great deal of data involving big samples and more regional areas are required for further study.

\section{References}

1. Cui Yan. Government roles in the pattern of home endowment[J]. Business Culture, 2008(8):101.

2. $\mathrm{Ru}$ Mengdan, Ji Huaxiong. City of a people-run non-enterprise unit endowment service function 
analysis[J] .Journal of Hulunbeier University,2015,2(23): 16-19.

3. Cheng Wei, Liu Haiying, Zhang Yuqi. Towards a Programme about Social Workers' Involvement in Home-Based Care Services for the Elderly in Community[J].Theory and Modernization,2013(1):58-61.

4. Zheng Shixiang. Thinking based on the aging society under the background of the arts students to participate in the home endowment services[J].Learning week,2015(5): 238.

5. Shi Wei, Xie Yu. The Urban Elderly' Choice of Home Care Providers and the Influencing Factors: From the Perspective of Welfare Pluralism[J]. Northwest Population Journal,2015,1(36): 48-54.

6. $\mathrm{Xu}$ Fengyun. Introduction to the cultivation of community elderly home care services volunteers[J].Seek Medical and Ask the Medicine,2012,10(7):614.

7. Maranta A, Sladowski PS (2010) The Changing Culture of Volunteering: A Skills-based Approach to Volunteer Involvement. A discussion paper by Volunteer Benevolves, Canada.

8. Yuan Xiaoxiao \, Keyonghong, Wu Minlun, et al. Probe into feasibility of application of university resources in community home care service[J]. Chinese Nursing Research,2013,27(6):1788.

9. Guo Guanjun, Wang Huimin, Wang Fuguo, et al. The old people life quality of yanji city and the present situation of old-age care demand that occupy the home[J].Journal of Qilu Nursing, 2015,21(6):56.

10. Gao Jing, Bai Dingxi, Wu Chenxi,et al. Investigation and study of the status and demand on home endowment service in four cities of sichuan province [J].Chinese Journal of Convalescent Medicine,2015,24(1): 92-95.

11. Zhao Rong, Tang Fengping, Li Ya.Survey of home aged care needs of urban elderly in Zhuzhou city and its countermeasure research[J]. Chinese Nursing Research,2015,29(4):136.

12. Xing Fengmei, Wang sudong, Wang Liwei,et al. Influencing factors of home care service demand in housebound elderly people[J].Chinese Journal of Public Health,2014,30(5):641-645.

13. Chen Zhike, Ma Shaozhen. The research of factors about the elderly demanding home care service-Based on the social investigation in Hunan Province[J].Journal of Central South University,2012,18(3):26-30.

14. McCallion, G.M.\& Stout, R.W.(1992).A comparison of community care of elderly people in Wales and Northern Ireland.Aging Clin. Exp. Res,4,348.

15. Wu Jialin, Wang Junqiao, Lu Meiling, et al. Care needs of community-dwelling adults aged 80 years and older: meeting of the needs[J].Journal of Nursing Science,2013,28(12):89-91. 